Pacific Journal of Mathematics

AN APPROACH TO SINGULAR HOMOLOGY THEORY 


\title{
AN APPROACH TO SINGULAR HOMOLOGY THEORY
}

\author{
TIBOR RADÓ
}

\section{IN TRODUCTION}

0.1. Given a topological space $X$, we associate with $X$ a complex $R=R(X)$ as follows. Let $E_{\infty}$ denote Hilbert space (that is, the space of all sequences $r_{1}, \cdots$, $r_{n}, \cdots$ of real numbers such that the series $r_{1}^{2}+\cdots+r_{n}^{2}+\cdots$ converges, with the usual definition of distance). For $p \geq 0$, let $v_{0}, \cdots, v_{p}$ be a sequence of $p+1$ points in $E_{\infty}$, which need not be linearly independent or distinct, and let $\left|v_{0}, \cdots, v_{p}\right|$ denote the convex hull of these points. Finally, let $T$ be a continuous mapping from $\left|v_{0}, \cdots, v_{p}\right|$ into $X$. Then the sequence $v_{0}, \cdots, v_{p}$ jointly with $T$ is a $p$-cell of the complex $R$, and will be denoted by $\left(v_{0}, \cdots\right.$, $\left.v_{p}, T\right)^{R}$. The group $C_{p}^{R}$ of (integral) $p$-chains in $R$ is defined as the free Abelian group with these $p$-cells as free generators. For $p<0, C_{p}^{R}$ is defined by $C_{p}^{R}=0$ (that is, $C_{p}^{R}$ consists then of a zero-element alone). The boundary operator $\partial_{p}^{R}$ : $C_{p}^{R} \rightarrow C_{p-1}^{R}$ is defined by the conventional formula

$$
\partial_{p}^{R}\left(v_{0}, \cdots, v_{p}, T\right)^{R}=\sum_{i=0}^{p}(-1)^{i}\left(v_{0}, \cdots, \hat{v}_{i}, \cdots, v_{p}, T\right)^{R}
$$

for $p \geq 1$. For $p \leq 0, \partial_{p}^{R}$ is defined as the trivial zero-homomorphism. Clearly $\partial \partial=0$, and thus $R=R(X)$ is a complex which is obviously closure-finite in the sense of [4]. Accordingly, one can define cycles $z_{p}^{R}$, boundaries $b_{p}^{R}$, and so forth, for $R$ in the usual manner. The homology groups of $R$ are defined by $H_{p}^{R}=$ $Z_{p}^{R} / B_{p}^{R}$, where $Z_{p}^{R}, B_{p}^{R}$ denote the group of $p$-cycles and $p$-boundaries respectively in $R$.

0.2 . The complex $R$, which was introduced and studied recently by the writer [6], differs from the various singular complexes used in previous literature first in the use of Hilbert space. The general practice is to consider continuous mappings $T$ from rectilinear simplexes located in any Euclidean space. Instead, we 
use Hilbert space in its capacity of infinite-dimensional Euclidean space, a procedure which may of course be adopted in all the various versions of singular homology theory. The main departure from previous practice lies however in the fact that no identifications are made in the chain groups $C_{p}^{R}$ of $R$ : two p-cells $\left(v_{0}^{\prime}, \cdots, v_{p}^{\prime}, T^{\prime}\right)^{R},\left(v_{0}^{\prime \prime}, \cdots, v_{p}^{\prime \prime}, T^{\prime \prime}\right)^{R}$ are equal if and only if they are identical, that is, if $v_{0}^{\prime}=v_{0}^{\prime \prime}, \cdots, v_{p}^{\prime}=v_{p}^{\prime \prime}, T^{\prime}=T^{\prime \prime}$. Thus the complex $R$ is of enormous size as compared with previously used complexes. Let us note that beyond the lack of identifications, $R$ is further increased by the fact that the points $v_{0}, \cdots, v_{p}$ occurring in a $p$-cell $\left(v_{0}, \cdots, v_{p}, T\right)^{R}$ are not required to be linearly independent or distinct.

0.3. There arises the question of how the homology groups of $R$ compare with those arising in previous approaches to singular homology theory. In [6], the writer proved that the homology groups of $R$ are isomorphic to those of the socalled total singular complex $S=S(X)$ introduced by Eilenberg [3]. Since this result will be used in the sequel, we shall now give the precise statement of the main theorem established in [6]. For each dimension $p \geq 0$, let us select a fundamental $p$-simplex, with (linearly independent) vertices $d_{0}, \cdots, d_{p}$. For our own purposes, it is convenient to choose $d_{0}, d_{1}, d_{2}, \cdots$ as the points $(1,0,0,0, \cdots)$, $(0,1,0,0, \cdots),(0,0,1,0, \cdots), \cdots$ in $E_{\infty}$. Given then a sequence $v_{0}, \cdots, v_{p}$ of $p+1$ points in $E_{\infty}$, which need not be linearly independent or distinct, there exists a unique linear map $\alpha:\left|d_{0}, \cdots, d_{p}\right| \rightarrow\left|v_{0}, \cdots, v_{p}\right|$ such that $\alpha\left(d_{i}\right)$ $=v_{i}, i=0, \cdots, p$. This linear map is denoted by $\left[v_{0}, \cdots, v_{p}\right]$. The total singular complex $S=S(X)$ of Eilenberg [3] may now be described as follows. For $p \geq 0$, a $p$-cell of $S$ is an aggregate $\left(d_{0}, \cdots, d_{p}, T\right)^{S}$, where $T$ is a continuous mapping from $\left|d_{0}, \cdots, d_{p}\right|$ into $X$. The group $C_{p}^{S}$ of (integral) $p$-chains of $S$ is then the free Abelian group with these p-cells as free generators. For $p<0$, one sets $C_{p}^{S}=0$. The boundary operator $\partial_{p}^{S}: C_{p}^{S} \rightarrow C_{p-1}^{S}$ is defined by

$$
\partial_{p}^{S}\left(d_{0}, \cdots, d_{p}, T\right)^{S}=\sum_{i=0}^{p}(-1)^{i}\left(d_{0}, \cdots, d_{p-1}, T\left[d_{0}, \cdots, \hat{d}_{i}, \cdots, d_{p}\right]\right)^{S},
$$

for $p \geq 1$. For $p \leq 0, \partial_{p}^{S}$ is the trivial zero-homomorphism. The homology groups of $S$ will be denoted by $H_{p}^{S}$. We have then obvious homomorphisms

$$
\sigma_{p}: C_{p}^{R} \rightarrow C_{p}^{S}, \quad \tau_{p}: C_{p}^{S} \rightarrow C_{p}^{R},
$$

defined as follows for $p \geq 0$ : 


$$
\begin{aligned}
& \tau_{p}\left(d_{0}, \cdots, d_{p}, T\right)^{S}=\left(d_{0}, \cdots, d_{p}, T\right)^{R}, \\
& \sigma_{p}\left(v_{0}, \cdots, v_{p}, T\right)^{R}=\left(d_{0}, \cdots, d_{p}, T\left[v_{0}, \cdots, v_{p}\right]\right)^{S} .
\end{aligned}
$$

For $p<0, \tau_{p}$ and $\sigma_{p}$ are defined as the trivial zero-homomorphisms. Unfortunately, $\tau_{p}$ is not a chain-mapping. On the other hand, $\sigma_{p}$ is easily seen to be a chainmapping, and hence it induces homomorphisms $\sigma_{* p}: H_{p}^{R} \rightarrow H_{p}^{S}$. The main result of [6] is contained in the following statement.

THEOREM. The homomorphism $\sigma_{* p}: H_{p}^{R} \rightarrow H_{p}^{S}$ is an isomorphism onto, for every dimension $p$.

Since singular homology theory is sometimes thought of only in relation to triangulable spaces, it may be appropriate to note that the preceding theorem is valid for general topological spaces. In particular, the space need not be arc-wise connected.

0.4 In view of the preceding theorem, the complex $R$ appears as an appropriate tool in constructing singular homology theory. It is of interest to note that the various complexes used in previous approaches to singular homology the ory may be derived from the complex $R$ by a combination of the following two types of reduction.

(i) The chain groups $C_{p}^{R}$ of $R$ are replaced by certain subgroups $\Gamma_{p}$. For example, one may select $\Gamma_{p}$ as the group generated by those $p$-cells $\left(v_{0}, \cdots\right.$, $\left.v_{p}, T\right)^{R}$ for which the points $v_{0}, \cdots, v_{p}$ are linearly independent. Another significant choice may be based upon the concept of a minimal complex studied by Eilenberg and Zilber [3].

(ii) One selects in $C_{p}^{R}$, for each $p$, a certain subgroup $G_{p}$, and one replaces $C_{p}^{R}$ by the factor group $C_{p}^{R} / G_{p}$ : From the computational point of view, this amounts to an identification of elements of $C_{p}^{R}$ which are contained in the same coset relative to $G_{p}$. For brevity, we shall refer to this type of process as an identification scheme.

In the present paper, we shall study the effect of the various identification schemes, occurring in previous theories, upon the homology structure of the complex $R$. It is easy to see that these identification schemes may be reduced to three basic types. Our result is that one may apply these basic identification schemes in any desired combination without changing the homology structure of $R$ (see Theorem 1 in \$4.7). As a matter of fact, we obtain an identification scheme which appears stronger than those previously used (see Theorem 2 in 4.7 and see 
$\S 5)$. This leads to some interesting questions, formulated in $\S 6$, which seem to deserve further study.

0.5. It should be noted that the complex $R$ is semisimplicial in the sense of [3], and therefore can be used to construct a complete homology and cohomology theory.

0.6. Previous relevant literature, as well as further problems arising in this line of thought, will be discussed in $\$ 6$ when convenient terminology will be available. The writer wishes to express his appreciation of the courtesy extended by S. Eilenberg and N. Steenrod who made available to him the manuscript of their yet unpublished book [2]. Both technically and conceptually, the study of that book proved most valuable in preparing the present paper.

\section{Identifigations in Mayer Complexes}

1.1. A Mayer complex $M$ is a collection of Abelian groups $C_{p}$, where the integer $p$ ranges from $-\infty$ to $+\infty$, together with homomorphisms

$$
\partial_{p}: C_{p} \rightarrow C_{p-1}
$$

such that $\partial_{p-1} \partial_{p}=0$. Cycles and boundaries are defined in the usual manner. The homology groups $H_{p}$ of $M$ are defined by $H_{p}=Z_{p} / B_{p}$, where $Z_{p}, B_{p}$ are the groups of $p$-cycles and $p$-boundaries respectively. If $M, M^{\prime}$ are Mayer complexes, then a set of homomorphisms

$$
f_{p}: C_{p} \rightarrow C_{p}^{\prime}
$$

is termed a chain mapping if $\partial_{p}^{\prime} f_{p}=f_{p-1} \partial_{p}$, where primes refer to the complex $M^{\prime}$

For clarity, we shall write $C_{p}^{M}, \partial_{p}^{M}, H_{p}^{M}$, and so on, to identify the complex under consideration. In particular, a $p$-chain of $M$ (that is, an element of $C_{p}^{M}$ ) will be denoted by symbols like $c_{p}^{M}, d_{p}^{M}$, and so forth.

1.2. We shall now describe the general process of identification in a Mayer complex $M$. Let $\left\{G_{p}\right\}$ be a collection of Abelian groups such that $G_{p} \subset C_{p}^{M}$ and

$$
\partial_{p}^{M} G_{p} \subset G_{p-1} .
$$

Explicitly: if $c_{p}^{M} \in G_{p}$, then $\partial_{p}^{M} c_{p}^{M} \in G_{p-1}$. Set $C_{p}^{m}=C_{p}^{M} / G_{p}$. Thus, the elements of $C_{p}^{m}$ are cosets selative to $G_{p}$. The general element of $C_{p}^{m}$ is of the 
form $\left\{c_{p}^{M}\right\}$, where this symbol denotes the coset containing the element $c_{p}^{M}$ of $C_{p}^{M}$. In view of (1), we can then define homomorphisms

$$
\partial_{p}^{m}: C_{p}^{m} \rightarrow C_{p-1}^{m}
$$

by the formula $\partial_{p}^{m}\left\{c_{p}^{M}\right\}=\left\{\partial_{p}^{M} c_{p}^{M}\right\}$. Clearly $\partial_{p-1}^{m} \partial_{p}^{m}=0$. Accordingly, the system of factor groups $\left\{C_{p}^{m}\right\}$, jointly with the homomorphisms $\partial_{p}^{m}$, constitutes a Mayer complex $m$. We shall say that $m$ is obtained by identification, with respect to the system $\left\{G_{p}\right\}$, from $M$. The system $\left\{G_{p}\right\}$, satisfying (1), will be termed an identifier for $M$. We have then natural homomorphisms

$$
\pi_{p}: C_{p}^{M} \rightarrow C_{p}^{m}
$$

defined by $\pi_{p} c_{p}^{M}=\left\{c_{p}^{M}\right\}$. Clearly

$$
\partial_{p}^{M} \pi_{p} c_{p}^{M}=\partial_{p}^{M}\left\{c_{p}^{M}\right\}=\left\{\partial_{p}^{M} c_{p}^{M}\right\}=\pi_{p-1} \partial_{p}^{M} c_{p}^{M} .
$$

Thus $\pi_{p}$ is a chain mapping, and hence induces homomorphisms

$$
\pi_{* p}: H_{p}^{M} \rightarrow H_{p}^{m}
$$

defined as follows. If $z_{p}^{M}$ is a cycle in $M$, then we let $\left[z_{p}^{M}\right]_{M}$ denote the homology class containing $z_{p}^{M}$. The symbol $\left[z_{p}^{m}\right]_{m}$ is defined similarly. Then $\pi_{* p}$ is given by

$$
\pi_{* p}\left[z_{p}^{M}\right]_{M}=\left[\pi_{p} z_{p}^{M}\right]_{m}
$$

If $\pi_{* p}$ is an isomorphism onto for every $p$, then we shall say that the identifier $\left\{G_{p}\right\}$ is unessential. Thus the process of identification with respect to an unessential identifier does not change the homology structure of the complex.

1.3. We shall state presently a convenient condition for the unessential character of an identifier $\left\{G_{p}\right\}$. Let us observe that the condition (1) in 1.2 means that the homomorphisms $\partial_{p}^{N}$, cut down to the subgroups $G_{p}$, may be used to turn the system $\left\{G_{p}\right\}$ into a Mayer complex which we call $G$. The complex $m$, defined in 1.2 , appears then as merely the complex $M \bmod G$ in the sense of the general relative homology theory of Mayer complexes. From this general theory, the condition for $\pi_{* p}$ to be an isomorphism onto, for all $p$, is well known: it is necessary and sufficient that all the homology groups of $G$ be trivial. For convenient application, we shall now state this condition explicitly.

The condition $(U)$. We shall say that the identifier $\left\{G_{p}\right\}$ satisfies the condition 
$(U)$ if the following holds: if $z_{p}^{M}$ is a cycle in $M$ such that $z_{p}^{M} \in G_{p}$ then there exists a $(p+1)$-chain $c_{p+1}^{M} \in G_{p+1}$ such that $\partial_{p+1}^{M} c_{p+1}^{M}=z_{p}^{M}$.

We have then the following criterion.

Criterion For UneSSential IDENTIFIERS. An identifier $\left\{G_{p}\right\}$ is unessential if and only if it satisfies condition $(U)$.

Since the elements of $G_{p}$ represent those elements of $C_{p}^{M}$ which are, in a sense, discarded as we pass from the complex $M$ to the complex $m$, the criterion may be also worded as follows: discarded cycles should bound discarded chains. In a special case, this criterion was used by Tucker [8]. As mentioned above, the general criterion is merely a re-wording of a well-known theorem in the relative homology theory of Mayer complexes (for a comprehensive presentation, see Eilenberg and Steenrod [2]). For the convenience of the reader, we shall now outline a direct proof of the criterion.

1.4. Assume first that the identifier $\left\{G_{p}\right\}$ is unessential. Take a cycle

$$
z_{p}^{M} \in G_{p} .
$$

Then $\pi_{p} z_{p}^{M}=\left\{z_{p}^{M}\right\}=0$, and hence $\pi_{* p}\left[z_{p}^{M}\right]_{M}=\left[\pi_{p} z_{p}^{M}\right]_{m}=0$. Since $\pi_{*}$ is an isomorphism onto, it follows that $z_{p}^{M}$ bounds in $M$ :

$$
z_{p}^{M}=\partial_{p+1}^{M} c_{p+1}^{M} \text {. }
$$

Application of $\pi_{p}$ yields, in view of (1), the equation

$$
0=\pi_{p} z_{p}^{M}=\pi_{p} \partial_{p+1}^{M} c_{p+1}^{M}=\partial_{p+1}^{M} \pi_{p+1} c_{p+1}^{M} \text {. }
$$

Thus $\pi_{p+1} c_{p+1}^{M}$ is a cycle of the complex $m$. Since $\pi_{*}$ is an isomorphism onto, we have therefore a cycle $z_{p+1}^{M}$ such that $\pi_{p+1} z_{p+1}^{M}$ differs from the cycle $\pi_{p+1} c_{p+1}^{M}$ only by a boundary. Thus we can write

$$
\pi_{p+1} z_{p+1}^{M}=\pi_{p+1} c_{p+1}^{M}+\partial_{p+2}^{m} c_{p+2}^{m} \text {. }
$$

Now $c_{p+2}^{m}$ is of the form $\left\{c_{p+2}^{M}\right\}=\pi_{p+2} c_{p+2}^{M}$. Making this substitution, we obtain

$$
\pi_{p+1} z_{p+1}^{M}=\pi_{p+1} c_{p+1}^{M}+\partial_{p+2}^{M} \pi_{p+2} c_{p+2}^{M}=\pi_{p+1}\left(c_{p+1}^{M}+\partial_{p+2}^{M} c_{p+2}^{M}\right) .
$$

Hence

$$
\pi_{p+1}\left(c_{p+1}^{M}-z_{p+1}^{M}+\partial_{p+2}^{M} c_{p+2}^{M}\right)=0
$$


Now let us consider the $(p+1)$-chain

$$
d_{p+1}^{M}=c_{p+1}^{M}-z_{p+1}^{M}+\partial_{p+2}^{M} c_{p+2}^{M} .
$$

By (3) we have $d_{p+1}^{M} \in G_{p+1}$, while from (2) we have $z_{p}^{M}=\partial_{p+1}^{M} d_{p+1}^{M}$. Thus (1) is seen to imply that $z_{p}^{M}$ bounds a chain contained in $G_{p+1}$. In other words, condition $(U)$ holds.

1.5. Assume now, conversely, that condition $(U)$ holds. We have to show that $\pi_{* p}$ is an isomorphism onto for every $p$.

(i) Suppose we have

$$
\pi_{* p}\left[z_{p}^{M}\right]_{M}=0
$$

for a certain cycle $z_{p}^{M}$. The assumption means that $\pi_{p} z_{p}^{M}$ bounds some chain $c_{p+1}^{m}$. Since $c_{p+1}^{m}$ is of the form $\left\{c_{p+1}^{M}\right\}=\pi_{p+1} c_{p+1}^{M}$, we have

$$
\pi_{p} z_{p}^{N}=\partial_{p+1}^{m} \pi_{p+1} c_{p+1}^{M}=\pi_{p} \partial_{p+1}^{M} c_{p+1}^{M}
$$

and hence

$$
\pi_{p}\left(z_{p}^{M}-\partial_{p+1}^{M} c_{p+1}^{M}\right)=0
$$

Thus the cycle

$$
Z_{p}^{M}=z_{p}^{M}-\partial_{p+1}^{M} c_{p+1}^{M}
$$

is contained in $G_{p}$. Since condition $(U)$ is now assumed, it follows that $Z_{p}^{M}$ is of the form

$$
Z_{p}^{M}=\partial_{p+1}^{M} d_{p+1}^{M}
$$

From (2) and (3) it follows that

$$
z_{p}^{M}=\partial_{p+1}^{M}\left(c_{p+1}^{M}+d_{p+1}^{M}\right) .
$$

Thus (1) implies that $z_{p}^{M}$ bounds in $M$, and hence $\pi_{* p}$ is an isomorphism into.

(ii) Assign now an element $\left[z_{p}^{m}\right]_{m}$ of $H_{p}^{m}$. Now $z_{p}^{m}$ is of the form

$$
z_{p}^{m}=\left\{c_{p}^{M}\right\}=\pi_{p} c_{p}^{M} .
$$

Since $z_{p}^{m}$ is a cycle, we have

$$
0=\partial_{p}^{m} z_{p}^{m}=\partial_{p}^{m} \pi_{p} c_{p}^{M}=\pi_{p-1} \partial_{p}^{M} c_{p}^{M}
$$


Hence

$$
\partial_{p}^{M} c_{p}^{M} \in G_{p-1} .
$$

Thus $\partial_{p}^{M} c_{p}^{M}$ is a cycle contained in $G_{p-1}$. Since condition $(U)$ is now assumed, we have a chain $d_{p}^{M}$ such that

$$
\partial_{p}^{M} c_{p}^{M}=\partial_{p}^{M} d_{p}^{M}
$$

Thus $c_{p}^{M}-d_{p}^{M}$ is a cycle:

$$
c_{p}^{M}-d_{p}^{M}=z_{p}^{M} .
$$

Now we calculate

$$
\pi_{* p}\left[z_{p}^{M}\right]_{M}=\left[\pi_{p} c_{p}^{M}-\pi_{p} d_{p}^{M}\right]_{m} .
$$

By (1), $\pi_{p} c_{p}^{M}=z_{p}^{m}$, and by (2), $\pi_{p} d_{p}^{M}=0$. Thus finally

$$
\pi_{* p}\left[z_{p}^{M}\right]_{M}=\left[z_{p}^{m}\right]_{m} .
$$

Thus $\pi_{* p}$ is onto, and the proof of the criterion is complete.

1.6. In marked contrast to the general character of the preceding discussion, the unessential identifiers actually employed in the sequel are of a very special and restricted type. There arises the question whether there are general constructions yielding unessential identifiers in Mayer complexes. The following comments may be of interest from this point of view. Let $M, L$ be Mayer complexes and let

$$
f_{p}: C_{p}^{M} \rightarrow C_{p}^{L}
$$

be a chain-mapping such that the induced homomorphisms $f_{* p}: H_{p}^{M} \rightarrow H_{p}^{L}$ are isomorphisms onto. In symbols:

$$
f_{* p}: H_{p}^{M} \approx H_{p}^{L}
$$

Let $N_{p}$ denote the nucleus of the homomorphism (1). Since $f_{p}$ is a chain-mapping, it is immediate that the system $\left\{N_{p}\right\}$ is an identifier.

In view of the strong assumption (2) one may be tempted to conjecture that $\left\{N_{p}\right\}$ is unessential. The following simple example shows that this is not the case, even under extremely special and favorable circumstances. Let $M$ be a finite simplicial complex described abstractly as follows. The group $C_{2}^{M}$ of (integral) 
2-chains of $M$ is the free Abelian group with a single generator $t$. The 1-chain group $C_{1}^{M}$ is the free Abelian group with four generators $s_{1}, s_{2}, s_{3}, s_{4}$. The 0 -chain group $C_{0}^{M}$ is generated by $a, b, c, d, e$. For $p \neq 0,1,2$, the $p$-chain group $C_{p}^{M}$ reduces to a zero-element. The boundary relations are as follows:

$$
\begin{gathered}
\partial t=s_{1}+s_{2}+s_{3}, \partial s_{1}=c-b, \partial s_{2}=a-c, \partial s_{3}=b-a, \partial s_{4}=e-d, \\
\partial a=\partial b=\partial c=\partial d=\partial e=0 .
\end{gathered}
$$

We define first homomorphisms $f_{p}: C_{p}^{M} \rightarrow C_{p}^{M}$ as follows:

$$
\begin{gathered}
f_{2} t=0, \quad f_{1} s_{1}=f_{1} s_{2}=f_{1} s_{3}=0, \quad f_{1} s_{4}=s_{1}+s_{2}+s_{3}, \\
f_{0} a=f_{0} b=f_{0} c=a, \quad f_{0} d=f_{0} e=d .
\end{gathered}
$$

For $p \neq 0,1,2$, of course $f_{p}$ is the trivial zero-homomorphism. Next we define homomorphisms $D_{p}: C_{p}^{M} \rightarrow C_{p+1}^{M}$ as follows:

$$
\begin{gathered}
D_{0} a=0, \quad D_{0} b=-s_{3}, \quad D_{0} c=s_{2}, \quad D_{0} d=0, \quad D_{0} e=-s_{4}, \\
D_{1} s_{1}=-t, \quad D_{1} s_{2}=0, \quad D_{1} s_{3}=0, \quad D_{1} s_{4}=t .
\end{gathered}
$$

For $p \neq 0,1$, of course $D_{p}$ is the trivial zero-homomorphism. One verifies readily the following facts.

(i) $f_{p}$ is a chain-mapping.

(ii) $\partial D_{p} \gamma_{p}^{M}+D_{p-1} \partial \gamma_{p}^{M}=f_{p} \gamma_{p}^{M}-\gamma_{p}^{M}$, for every $p$-chain $\gamma_{p}^{M}$ of $M$. Thus $f_{p} \simeq 1$.

(iii) Let $N_{p}$ be the nucleus of $f_{p}$, and let $m$ be the complex obtained from $M$ by using the identifier $\left\{N_{p}\right\}$, in the sense of 1.2. Then the 1-dimensional homology group $H_{1}^{m}$ of $m$ is infinite cyclic.

(iv) The 1-dimensional homology group $H_{1}^{M}$ of $M$ is trivial (consists of zero alone).

Thus $M$ and $m$ have different homology structures, and hence $\left\{N_{p}\right\}$ is certainly not unessential. And yet, in view of (i), (ii), the induced homomorphisms $f_{* p}$ : $H_{p}^{M} \rightarrow H_{p}^{M}$ are isomorphisms onto. In other words, a very plausible method to obtain unessential identifiers fails even under very special and favorable conditions.

1.7. In dealing with additively written Abelian groups, we shall use certain familiar conventions. Thus we shall write $G=0$ to state that the Abelian group $G$ 
is trivial (consists of a zero-element alone). If $A_{1}, \cdots, A_{n}$ are subgroups of $G$, then $A_{1}+\cdots+A_{n}$ will denote the smallest subgroup containing $A_{1}, \cdots, A_{2}$.

\section{The Auxiliary Complex $K$}

2.1. The auxiliary complex $K$, which played an important role in [6] already, is merely the "formal complex," in the sense of [2], of $E_{\infty}$ taken as a point set. The complex $K$ is defined as follows. For $p \geq 0$, a $p$-cell of $K$ is a sequence $\left(v_{0}, \cdots, v_{p}\right)$ of points of $E_{\infty}$ which are not required to be linearly independent or distinct. Two $p$-cells $\left(v_{0}, \cdots, v_{p}\right),\left(w_{0}, \cdots, w_{p}\right)$ are considered as equal if and only if $v_{i}=w_{i}, i=0, \cdots, p$. These $p$-cells are taken as a base for a free Abelian group, to be denoted by $C_{p}$, the group of (finite) $p$-chains of $K$. For $p<0$, one defines $C_{p}=0$. For $p \geq 1$, the boundary operator

$$
\partial_{p}: C_{p} \rightarrow C_{p-1}
$$

is defined by the formula

$$
\partial_{p}\left(v_{0}, \cdots, v_{p}\right)=\sum_{i=0}^{p}(-1)^{i}\left(v_{0}, \cdots, \hat{v}_{i}, \cdots, v_{p}\right)
$$

Clearly $\partial \partial=0$. For $p \leq 0, \partial_{p}$ is of course defined as the trivial zero homomorphism.

Let $\left(v_{0}, \cdots, v_{p}\right)$ be a $p$-cell of $K$ : Treating the points of $E_{\infty}$ as vectors in the usual manner, we describe the barycenter $b=b\left(v_{0}, \cdots, v_{p}\right)$ of the points $v_{0}$, $\cdots, v_{p}$ by the formula

$$
b=\frac{v_{0}+\cdots+v_{p}}{p+1} .
$$

2.2. The following homomorphisms will be used.

(i) The homomorphism $\partial_{p}: C_{p} \rightarrow C_{p-1}$, already defined.

(ii) In terms of any assigned point $v$ of $E_{\infty}$, one defines the cone homomorphism

$$
h_{p}^{v}: C_{p} \rightarrow C_{p+1}
$$

by the formula

$$
h_{p}^{v}\left(v_{0}, \cdots, v_{p}\right)=(-1)^{p+1}\left(v_{0}, \cdots, v_{p}, v\right) .
$$


For $p<0, h_{p}^{v}$ is the trivial zero homomorphism.

(iii) The barycentric homomorphism

$$
\beta_{p}: C_{p} \rightarrow C_{p}
$$

is defined as follows. For $p<0, \beta_{p}$ is the trivial zero homomorphism. For $p=0$, $\beta_{0}=1$, the identity. For $p>1, \beta_{p}$ is defined recursively by the formula

$$
\beta_{p}\left(v_{0}, \cdots, v_{p}\right)=h_{p-1}^{b} \beta_{p-1} \partial_{p}\left(v_{0}, \cdots, v_{p}\right),
$$

where $b$ is the barycenter of the points $v_{0}, \cdots, v_{1}$.

(iv) The barycentric homotopy operator

$$
\rho_{p}: C_{p} \longrightarrow C_{p+1}
$$

is defined as follows. For $p \leq 0, \rho_{p}\left(v_{0}, \cdots, v_{p}\right)=0$. For $p \geq 1, \rho_{p}$ is defined recursively by the formula

$$
\rho_{p}\left(v_{0}, \cdots, v_{p}\right)=h_{p}^{b}\left(\beta_{p}-1-\rho_{p-1} \partial_{p}\right)\left(v_{0}, \cdots, v_{p}\right),
$$

where $b$ is the barycenter of the points $\left(v_{0}, \cdots, v_{p}\right)$.

(v) For $p \geq 1,0 \leq j \leq p-1$, we define the homomorphism.

$$
t_{p, j}: C_{p} \longrightarrow C_{p}
$$

by the formula $t_{p, j}\left(v_{0}, \cdots, v_{j}, v_{j+1}, \cdots, v_{p}\right)=\left(v_{0}, \cdots, v_{j+1}, v_{j}, \cdots, v_{p}\right)$. The operation $t_{p, j}$ will be referred to as a transposition. Thus "transposition" means here a transposition of adjacent elements. According to the definition of equality for $p$-cells (see 2.1), we have $t_{p, j}\left(v_{0}, \cdots, v_{p}\right)=\left(v_{0}, \cdots, v_{p}\right)$ if and only if $v_{j}=v_{j+1}$.

2.3. The following identities hold among these various homomorphisms

(i) $\partial_{p+1} h_{p}^{v}+h_{p-1}^{v} \partial_{p}=1$

(ii) $\partial_{p} \beta_{p}=\beta_{p-1} \partial_{p}$,

(iii) $\partial_{p+1} \rho_{p}+\rho_{p-1} \partial_{p}=\beta_{p}-1$,

(iv) $\beta_{p} t_{p, j}=-\beta_{p}$

$$
(p \geq 1, \quad 0 \leq j \leq p-1) .
$$

2.4. If $\left(v_{0}, \cdots, v_{p}\right)$ is a $p$-cell of $K$, then $\left|v_{0}, \cdots, v_{p}\right|$ denotes the convex hull of the points $v_{0}, \cdots, v_{p}$ (that is, the smallest convex set containing these 
points). If $c_{p}$ is a $p$-chain of $K$, and $A$ is a convex set in $E_{\infty}$, then the inclusion $c_{p} \subset A$ is defined to mean that $c_{p}$ can be written in the form

$$
c_{p}=\sum_{j=1}^{n} k_{j}\left(v_{0, j}, \cdots, v_{p, j}\right) \text {, }
$$

where the coefficients $k_{j}$ are of course integers, so that $\left|v_{0, j}, \cdots, v_{p, j}\right| \subset A$, $j=1, \cdots, n$. One has then the following inclusions:

$$
\begin{aligned}
& \partial_{p}\left(v_{0}, \cdots, v_{p}\right) \subset\left|v_{0}, \cdots, v_{p}\right|, \\
& \beta_{p}\left(v_{0}, \cdots, v_{p}\right) \subset\left|v_{0}, \cdots, v_{p}\right|, \\
& \rho_{p}\left(v_{0}, \cdots, v_{p}\right) \subset\left|v_{0}, \cdots, v_{p}\right|, \\
& t_{p, j}\left(v_{0}, \cdots, v_{p}\right) \subset\left|v_{0}, \cdots, v_{p}\right| .
\end{aligned}
$$

As a consequence, an inclusion $c_{p} \subset A$ implies that $\partial_{p} c_{p} \subset A, \beta_{p} c_{p} \subset A$, $\rho_{p} c_{p} \subset A, t_{p, j} c_{p} \subset A$. It is understood that the zero chain $c_{p}=0$ is agreed to satisfy the inclusion $c_{p} \subset A$ for every convex set $A$.

2.5. For $p \geq 1$, an elementary $t$-chain in $K$ is defined as a $p$-chain $c_{p}$ which can be written in the form (see $2.2(\mathrm{v}))$

$$
c_{p}=\left(v_{0}, \cdots, v_{p}\right)+t_{p, j}\left(v_{0}, \cdots, v_{p}\right)
$$

LEMMA. Given an elementary t-chain

$$
c_{p}=\left(v_{0}, \cdots, v_{p}\right)+t_{p, j}\left(v_{0}, \cdots, v_{p}\right) \quad(p \geq 1),
$$

the following statements hold:

(i) If $p=1$, then $\partial_{p} c_{p}=0$. If $p>1$, then $\partial_{p} c_{p}$ is a linear combination (with integral coefficients) of elementary t-chains $\subset\left|v_{0}, \cdots, v_{p}\right|$;

(ii) $\beta_{p} c_{p}=0$;

(iii) $\rho_{p} c_{p}$ is a linear combination (with integral coefficients) of elementary t-chains $\subset\left|v_{0}, \cdots, v_{p}\right|$.

Proof. The assertion (ii) is an immediate consequence of 2.4 (iv). The assertions (i) and (iii) are readily verified for $p=1$. Hence we can assume that $p>1$.

Proof of (i) for $p>1$. Lét us note that $t_{p, j}\left(v_{0}, \cdots, v_{p}\right)$ is of the form $\left(w_{0}\right.$, $\left.\cdots, w_{p}\right)$, where $v_{i}=w_{i}$ for $i \neq j, j+1$, and $v_{j}=w_{j+1}, v_{j+1}=w_{j}$. Now we have 


$$
\partial_{p} c_{p}=\sum_{i=0}^{p}(-1)^{i}\left[\left(v_{0}, \cdots, \hat{v}_{i}, \cdots, v_{p}\right)+\left(w_{0}, \cdots, \hat{w}_{i}, \cdots, w_{p}\right)\right] .
$$

For $i \neq j, j+1$, the quantity in square brackets is clearly an elementary $t$-chain $\subset\left|v_{0}, \cdots, v_{p}\right|$. On the other hand, the terms corresponding to $i=j$ and $i=j+1$ cancel. Thus (i) follows.

Proof of (iii) for $p>1$. Since (iii) is verified directly for $p=1$, we proceed by induction. Assume (iii) to hold for $p-1$, where $p \geq 2$. Let us write again $t_{p, j}=\left(w_{0}, \cdots, w_{p}\right)$. Clearly, the points $v_{0}, \cdots, v_{p}$ and the points $w_{0}, \cdots, w_{p}$ have the same barycenter $b$. Hence we have (see 2.2 (iv))

$$
\begin{aligned}
\rho_{p}\left(v_{0}, \cdots, v_{p}\right)=h_{p}^{b}\left[\beta_{p}\left(v_{0}, \cdots, v_{p}\right)\right. & -\left(v_{0}, \cdots, v_{p}\right) \\
& \left.-\rho_{p-1} \partial_{p}\left(v_{0}, \cdots, v_{p}\right)\right], \\
\rho_{p} t_{p, j}\left(v_{0}, \cdots, v_{p}\right)=h_{p}^{b}\left[\beta_{p} t_{p, j}\left(v_{0}, \cdots, v_{p}\right)\right. & -t_{p, j}\left(v_{0}, \cdots, v_{p}\right) \\
& \left.-\rho_{p-1} \partial_{p} t_{p, j}\left(v_{0}, \cdots, v_{p}\right)\right] .
\end{aligned}
$$

In view of (ii), addition yields

$$
\rho_{p} c_{p}=h_{p}^{b}\left(-c_{p}-\rho_{p-1} \partial_{p} c_{p}\right) \text {. }
$$

Now, by (i), $\partial_{p} c_{p}$ is a linear combination (with integral coefficients) of elementary $t$-chains $\subset\left|v_{0}, \cdots, v_{p}\right|$. Hence, by the inductive assumption, the same holds for $\rho_{p-1} \partial_{p} c_{p}$, and hence also for the quantity in parentheses in (1), and finally for $\rho_{p} c_{p}$ itself, since $b \in\left|v_{0}, \cdots, v_{p}\right|$.

2.6. For $p \geq 1$, an elementary $d$-chain in $K$ is defined as a $p$-cell $\left(v_{0}, \cdots, v_{p}\right)$ such that $v_{j}=v_{j+1}$ for some $j$.

LeммA. If $c_{p}=\left(v_{0}, \cdots, v_{p}\right)$ is an elementary $d$-chain, then the following statements hold.

(i) If $p=1$, then $\partial_{p} c_{p}=0$. If $p>1$, then $\partial_{p} c_{p}$ is a linear combination (with integral coefficients) of elementary $d$-chains $\subset\left|v_{0}, \cdots, v_{p}\right|$.

(ii) $\beta_{p} c_{p}=0$.

(iii) $\rho_{p} c_{p}$ is a linear combination (with integral coefficients) of elementary d-chains $\subset\left|v_{0}, \cdots, v_{p}\right|$.

The proof is entirely analogous to that in 2.5 , except that (ii) requires an additional remark. We have $v_{j}=v_{j+1}$ for some $j$ by assumption. For this same $j$, 
we have then the relation

$$
t_{p, j}\left(v_{0}, \cdots, v_{p}\right)=\left(v_{0}, \cdots, v_{p}\right) .
$$

Hence we have also

$$
\beta_{p} t_{p, j}\left(v_{0}, \cdots, v_{p}\right)=\beta_{p}\left(v_{0}, \cdots, v_{p}\right) .
$$

On the other hand, 2.3 (iv) yields

$$
\beta_{p} t_{p, j}\left(v_{0}, \cdots, v_{p}\right)=-\beta_{p}\left(v_{0}, \cdots, v_{p}\right) .
$$

Hence $2 \beta_{p}\left(v_{0}, \cdots, v_{p}\right)=0$. Since $\beta_{p}\left(v_{0}, \cdots, v_{p}\right)$ is an element of the free Abelian group $C_{p}$, it follows that $\beta_{p}\left(v_{0}, \cdots, v_{p}\right)=0$.

\section{The Complex $R=R(X)$}

3.1. In working with the complex $R$ (see 0.1 ), the following device (introduced by Eilenberg and Steenrod in [2] in connection with the complex $S$; see 0.3 ) is useful. Let $A$ be a convex subset of $E_{\infty}$, and let $C_{p}^{A}$ denote the subgroup of $C_{p}$ (see 2.1) generated by those $p$-cells $\left(v_{0}, \cdots, v_{p}\right)$ of the complex $K$ which satisfy the inclusion $\left(v_{0}, \cdots, v_{p}\right) \subset A$ (see 2.4). For $p<0$, we define $C_{p}^{A}=0$ (see 1.7). Let $T: A \rightarrow X$ be a continuous mapping. We can define then homomorphisms

$$
T_{p}: C_{p}^{A} \rightarrow C_{p}^{R}
$$

by the formula

$$
T_{p}\left(v_{0}, \cdots, v_{p}\right)=\left(v_{0}, \cdots, v_{p}, T\right)^{R} \quad\left(p \geq 0,\left(v_{0}, \cdots, v_{p}\right) \subset C_{p}^{A}\right) .
$$

For $p<0, T_{p}$ is the trivial zero-homomorphism. For $c_{p} \in C_{p}^{A}$, it will be convenient to use the symbol $\left(c_{p}, T\right)^{R}$ to denote $T_{p} c_{p}$. Among the simple and obvious rules of computation for the symbol $\left(c_{p}, T\right)^{R}$, we mention the formula

$$
\partial_{p}^{R}\left(c_{p}, T\right)^{R}=\left(\partial_{p} c_{p}, T\right)^{R} .
$$

In terms of the preceding notations, we define now homomorphisms

$$
\begin{gathered}
\beta_{p}^{R}: \quad C_{p}^{R} \rightarrow C_{p}^{R}, \\
\rho_{p}^{R}: \quad C_{p}^{R} \longrightarrow C_{p+1}^{R}
\end{gathered}
$$


by the formulas

$$
\begin{array}{ll}
\beta_{p}^{R}\left(v_{0}, \cdots, v_{p}, T\right)^{R}=\left(\beta_{p}\left(v_{0}, \cdots, v_{p}\right), T\right)^{R}, & (p \geq 0), \\
\rho_{p}^{R}\left(v_{0}, \cdots, v_{p}, T\right)^{R}=\left(\rho_{p}\left(v_{0}, \cdots, v_{p}\right), T\right)^{R} & (p \geq 0) .
\end{array}
$$

Since $\beta_{p}\left(v_{0}, \cdots, v_{p}\right) \subset\left|v_{0}, \cdots, v_{p}\right|, \rho_{p}\left(v_{0}, \cdots, v_{p}\right) \subset\left|v_{0}, \cdots, v_{p}\right|$ by 2.4 , the homomorphisms $\beta_{p}^{R}, \rho_{p}^{R}$ are well defined. For $p<0, \beta_{p}^{R}$ and $\rho_{p}^{R}$ are defined as the trivial zero homomorphisms. In terms of the homomorphisms $t_{p, j}$ defined in 2.2 , we define

$$
t_{p, j}^{R}: C_{p}^{R} \rightarrow C_{p}^{R}, \quad(p \geq 1, \quad 0 \leq j \leq p-1),
$$

by means of the formula

$$
t_{p, j}^{R}\left(v_{0}, \cdots, v_{p}, T\right)^{R}=\left(t_{p, j}\left(v_{0}, \cdots, v_{p}\right), T\right)^{R} .
$$

We have then the following identities (see [6]):

$$
\begin{gathered}
\partial_{p}^{R} \beta_{p}^{R}=\beta_{p-1}^{R} \partial_{p}^{R} ; \\
\partial_{p+1}^{R} \rho_{p}^{R}+\rho_{p-1}^{R} \partial_{p}^{R}=\beta_{p}^{R}-1,
\end{gathered}
$$

where 1 denotes the identity transformation in $C_{p}^{R}$; furthermore (see 0.3 )

$$
\beta_{p}^{R} t_{p, j}^{R}=-\beta_{p}^{R}
$$

(4)

$$
\sigma_{p} \tau_{p}=1 \text {; }
$$$$
\partial_{p}^{S}=\sigma_{p-1} \partial_{p}^{R} \tau_{p}
$$$$
\sigma_{p-1} \partial_{p}^{R} \tau_{p} \sigma_{p}=\sigma_{p-1} \partial_{p}^{R}
$$$$
\sigma_{p} \beta_{p}^{R} \tau_{p} \sigma_{p}=\sigma_{p} \beta_{p}^{R} \text {; }
$$

$\sigma_{p+1} \rho_{p}^{R} \tau_{p} \sigma_{p}=\sigma_{p+1} \rho_{p}^{R}$;

$\tau_{p-1} \sigma_{p-1} \partial_{p}^{R} \beta_{p}^{R}=\partial_{p}^{R} \tau_{p} \sigma_{p} \beta_{p}^{R}$.

3.2. For $p \geq 1$, we define an elementary $t$-chain in $R$ as a chain of the form $\left(v_{0}, \cdots, v_{p}, T\right)^{R}+\left(t_{p, j}\left(v_{0}, \cdots, v_{p}\right), T\right)^{R}$ (see 2.2). The subgroup of $C_{p}^{R}$ generated by the elementary $t$-chains will be denoted by $T_{p}^{R}$. For $p \leq 0$, we define $T_{p}^{R}=0$. 
Lemma. If $c_{p}^{R} \in T_{p}^{R}$, then

$$
\begin{aligned}
& \partial_{p}^{R} c_{p}^{R} \in T_{p-1}^{R}, \\
& \beta_{p}^{R} c_{p}^{R}=0
\end{aligned}
$$$$
\rho_{p}^{R} c_{p}^{R} \in T_{p+1}^{R}
$$

Proof. Clearly, it is sufficient to consider the case where $c_{p}^{R}$ is an elementary $t$-chain:

$$
\begin{aligned}
c_{p}^{R}=\left(v_{0}, \cdots, v_{p}, T\right)^{R}+\left(t_{p, j}\left(v_{0}, \cdots, v_{p}\right), T\right)^{R} & \\
& =\left(\left(v_{0}, \cdots, v_{p}\right)+t_{p, j}\left(v_{0}, \cdots, v_{p}\right), T\right)^{R} .
\end{aligned}
$$

Then we have

$$
\partial_{p}^{R} c_{p}^{R}=\left(\partial_{p}\left[\left(v_{0}, \cdots, v_{p}\right)+t_{p, j}\left(v_{0}, \cdots, v_{p}\right)\right], T\right)^{R} .
$$

By 2.5 (i), $\partial_{p}\left[\left(v_{0}, \cdots, v_{p}\right)+t_{p, j}\left(v_{0}, \cdots, v_{p}\right)\right]$ is either zero or else a linear combination, with integral coefficients, of $(p-1)$-chains of the form $\left(w_{0}, \cdots\right.$, $\left.w_{p-1}\right)+t_{p-1, n}\left(w_{0}, \cdots, w_{p-1}\right)$, all $\subset\left|v_{0}, \cdots, v_{p}\right|$, and thus (i) is obvious. In a similar manner, (ii) and (iii) follow from 2.5 (ii) and 2.5 (iii).

3.3. For $p \geq 1$, we define an elementary $d$-chain in $R$ as a $p$-cell $\left(v_{0}, \cdots\right.$, $\left.v_{p}, T\right)^{R}$ such that $v_{j}=v_{j+1}$ for some $j, 0 \leq j \leq p-1$. The subgroup of $C_{p}^{R}$ generated by the elementary $d$-chains is denoted by $D_{p}^{R}$. For $p \leq 0$, we define $D_{p}^{R}=0$.

Lemma. If $c_{p}^{R} \in D_{p}^{R}$, then

$$
\begin{aligned}
& \partial_{p}^{R} c_{p}^{R} \in D_{p-1}^{R}, \\
& \beta_{p}^{R} c_{p}^{R}=0, \\
& \rho_{p}^{R} c_{p}^{R} \in D_{p+1}^{R} .
\end{aligned}
$$

These statements are immediate consequences of 2.6 (i), 2.6 (ii), 2.6 (iii).

3.4 Given a $p$-cell $\left(v_{0}, \cdots, v_{p}, T\right)^{R}$, take a sequence $w_{0}, \cdots, w_{p}$ of $p+1$ linearly independent points in $E_{\infty}$. Then we have a linear mapping $\alpha: \mid w_{0}, \cdots$, $w_{p}|\rightarrow| v_{0}, \cdots, v_{p} \mid$ such that $\alpha\left(w_{i}\right)=v_{i}, i=0, \cdots, p$. Then the $p$-chain

$$
c_{p}^{R}=\left(v_{0}, \cdots, v_{p}, T\right)^{R}-\left(w_{0}, \cdots, w_{p}, T \alpha\right)^{R}
$$


will be termed an elementary a-chain. The subgroup of $C_{p}^{R}$ generated by the elementary a-chains will be denoted by $A_{p}^{R}$. For $p<0$, we define $A_{p}^{R}=0$.

LeмmA. $c_{p}^{R} \in A_{p}^{R}$ if and only if $\sigma_{p} c_{p}^{R}=0$ (see 0.3).

Proof. Assume $c_{p}^{R} \in A_{p}^{R}$. Then $c_{p}^{R}$ is a linear combination of chains of the form (1), and hence it is sufficient to show that $\sigma_{p} c_{p}^{R}=0$ for the chain (1). Now we have (see 0.3)

$$
\sigma_{p} c_{p}^{R}=\left(d_{0}, \cdots, d_{p}, T\left[v_{0}, \cdots, v_{p}\right]\right)^{S}-\left(d_{0}, \cdots, d_{p}, T \alpha\left[w_{0}, \cdots, w_{p}\right]\right)^{S} .
$$

Clearly $\left[v_{0}, \cdots, v_{p}\right]=\alpha\left[w_{0}, \cdots, w_{p}\right]$, and thus $\sigma_{p} c_{p}^{R}=0$.

Assume next that $\sigma_{p} c_{p}^{R}=0$. Then we also have $\tau_{p} \sigma_{p} c_{p}^{R}=0$. The chain $c_{p}^{R}$ can be written as a (finite) sum

$$
c_{p}^{R}=\sum_{j} n_{j}\left(v_{0, j}, \cdots, v_{p, j}, T_{j}\right)^{R}
$$

where the coefficients $n_{j}$ are integers. We have then

$$
0=\tau_{p} \sigma_{p} c_{p}^{R}=\sum_{j} n_{j}\left(d_{0}, \cdots, d_{p}, T_{j}\left[v_{0, j}, \cdots, v_{p, j}\right]\right)^{R}
$$

Subtracting (3) from (2), we see that $c_{p}^{R}$ appears as a linear combination of elementary a-chains, and thus $c_{p}^{R} \in A_{p}^{R}$. If $p<0$, then the lemma is of course obvious.

3.5. Lemma. If $c_{p}^{R} \in A_{p}^{R}$, then

$$
\begin{aligned}
& \partial_{p}^{R} c_{p}^{R} \in A_{p-1}^{R}, \\
& \beta_{p}^{R} c_{p}^{R} \in A_{p}^{R}, \\
& \rho_{p}^{R} c_{p}^{R} \in A_{p+1}^{R} .
\end{aligned}
$$

These statements are immediate consequences of the identities (6), (7), (8) in 3.1 , in connection with the lemma in 3.4 . For example, to prove (iii), we note that by (8) in 3.1 , we have

$$
\sigma_{p+1} \rho_{p}^{R} c_{p}^{R}=\sigma_{p+1} \rho_{p}^{R} \tau_{p} \sigma_{p} c_{p}^{R}=0,
$$

since $c_{p}^{R} \in A_{p}^{R}$, and hence $\sigma_{p} c_{p}^{R}=0$ by 3.4. Also by 3.4, the relation (1) implies 
that $\rho_{p}^{R} c_{p}^{R} \in A_{p+1}^{R}$.

3.6. Let us observe that the chain groups $C_{p}^{R}, C_{p}^{S}$ are free Abelian groups by their very definition (see 0.3 ) and hence they do not contain elements of finite order.

4. UNESSENTIA L IDENTIF IG A TIONS IN $R=R(X)$

4.1. Lemma. Let $\left\{G_{p}\right\}$ be an identifier for $R$ (see 1.2, 0.1 ) such that the following conditions hold:

$$
\begin{aligned}
& c_{p}^{R} \in G_{p} \text { implies that } \beta_{p}^{R} c_{p}^{R}=0 ; \\
& c_{p}^{R} \in G_{p} \text { implies that } \rho_{p}^{R} c_{p}^{R} \in G_{p}+1 .
\end{aligned}
$$

Then $\left\{G_{p}\right\}$ is unessential (see 1.2).

Proof. We shall verify that $\left\{G_{p}\right\}$ satisfies condition $(U)$ of 1.3 . Take a cycle $z_{p}^{R} \in G_{p}$. In view of (i) and (ii), the homotopy identity

$$
\partial_{p+1}^{R} \rho_{p}^{R} z_{p}^{R}+\rho_{p-1}^{R} \partial_{p}^{R} z_{p}^{R}=\beta_{p}^{R} z_{p}^{R}-z_{p}^{R}
$$

yields the relation

$$
z_{p}^{R}=-\partial_{p+1}^{R} \rho_{p}^{R} z_{p}^{R} .
$$

Thus $z_{p}^{R}$ is the boundary of the $(p+1)$-chain $\rho_{p}^{R} z_{p}^{R} \in G_{p+1}$, and condition $(U)$ is established. By the criterion in 1.3, it follows that $\left\{G_{p}\right\}$ is unessential.

4.2. Lemma. Let $\left\{G_{p}\right\}$ be an identifier for $R$, such that the following conditions hold:

$$
G_{p} \supset A_{p}^{R}(\text { see 3.4); }
$$$$
c_{p}^{R} \in G_{p} \text { implies that } \sigma_{p} \beta_{p}^{R} c_{p}^{R}=0 \text { (see 0.3); }
$$$$
c_{p}^{R} \in G_{p} \text { implies that } \rho_{p}^{R} c_{p}^{R} \in G_{p+1} \text {. }
$$

Then $\left\{G_{p}\right\}$ is unessential.

Proof. Again, we verify that $\left\{G_{p}\right\}$ satisfies condition $(U)$. Let us take a cycle $z_{p}^{R} \in G_{p}$; we have to show that it is the boundary of some chain in $G_{p+1}$. We note that 
(1)

$$
\zeta_{p}^{R}=\beta_{p}^{R} z_{p}^{R}
$$

is a cycle, and that by (ii) we have

$$
\sigma_{p} \zeta_{p}^{R}=\sigma_{p} \beta_{p}^{R} z_{p}^{R}=0
$$

since $z_{p}^{R} \in G_{p}$. Since $\sigma_{* p}: H_{p}^{R} \rightarrow H_{p}^{S}$ is an isomorphism onto (see 0.3 ), it follows that $\zeta_{p}^{R}$ bounds:

$$
\zeta_{p}^{R}=\partial_{p+1}^{R} \gamma_{p+1}^{R}
$$

Applying $\sigma_{p}$ on the left, we get (see 0.3 )

$$
0=\sigma_{p} \zeta_{p}^{R}=\sigma_{p} \partial_{p+1}^{R} \gamma_{p+1}^{R}=\partial_{p+1}^{S} \sigma_{p+1} \gamma_{p+1}^{R}
$$

Thus $\sigma_{p+1} \gamma_{p+1}^{R}$ is a cycle:

$$
\sigma_{p+1} \gamma_{p+1}^{R}=z_{p+1}^{S}
$$

Since $\sigma_{*}$ is an isomorphism onto (see 0.3 ), there exists a cycle $z_{p+1}^{R}$ such that $z_{p+1}^{S}$ and $\sigma_{p+1} z_{p+1}^{R}$ differ only in a boundary:

$$
z_{p+1}^{S}=\sigma_{p+1} z_{p+1}^{R}+\partial_{p+2}^{S} c_{p+2}^{S} .
$$

Since $\partial_{p+2}^{S}=\sigma_{p+1} \partial_{p+2}^{R} \tau_{p+2}$, the relations (3) and (4) yield

$$
\sigma_{p+1}\left(\gamma_{p+1}^{R}-z_{p+1}^{R}-\partial_{p+2}^{R} \tau_{p+2} c_{p+2}^{S}\right)=0 .
$$

On setting

$$
d_{p+1}^{R}=\gamma_{p+1}^{R}-z_{p+1}^{R}-\partial_{p+2}^{R} \tau_{p+2} c_{p+2}^{S},
$$

we see that the relations (5), (1), (2), (6) yield

$$
\begin{gathered}
\sigma_{p+1} d_{p+1}^{R}=0, \\
\beta_{p}^{R} z_{p}^{R}=\partial_{p+1}^{R} d_{p+1}^{R} .
\end{gathered}
$$

From the homotopy identity 4.1 (1) and from (8) we infer now that

$$
z_{p}^{R}=\partial_{p+1}^{R}\left(d_{p+1}^{R}-\rho_{p}^{R} z_{p}^{R}\right) \text {. }
$$

By (7), (i), and 3.4, we have $d_{p+1}^{R} \in G_{p+1}$. Since $\rho_{p}^{R} z_{p}^{R} \in G_{p+1}$ by (iii), it follows from (9) that $z_{p}^{R}$ is the boundary of a chain in $G_{p+1}$, and the proof of the 
lemma is complete,

4.3. LемMA. Let $\left\{G_{p}\right\}$ be an identifier for $R$ which satisfies the assumptions of the lemma in 4.1. For each $p$, let $\hat{G}_{p}$ denote the division-hull of $G_{p}$. Then $\left\{\hat{G}_{p}\right\}$ is again an identifier (see 1.2) which satisfies the assumptions of Lemma 4.1.

Proof. Take a chain $c_{p}^{R} \in \hat{G}_{p}$. Then there exists an integer $n \neq 0$, such that $n c_{p}^{R} \in G_{p}$ and hence (since $\left\{G_{p}\right\}$ satisfies the assumptions of Lemma 4.1)

$$
\begin{aligned}
& n \beta_{p}^{R} c_{p}^{R}=0, \\
& n \rho_{p}^{R} c_{p}^{R} \in G_{p+1} .
\end{aligned}
$$

By the definition of $\hat{G}_{p+1}$, (2) implies that $\rho_{p}^{R} c_{p}^{R} \in \hat{G}_{p+1}$. Since $\beta_{p}^{R} c_{p}^{R}$ is an element of the free Abelian group $C_{p}^{R}$ (see 3.6), (1) implies that $\beta_{p}^{R} c_{p}^{R}=0$.

4.4. LEMMA. Let $\left\{G_{p}\right\}$ be an identifier for $R$ which satisfies the assumptions of Lemma 4.2. Then $\left\{\hat{G}_{p}\right\}$ is again an identifier which satisfies the assumptions of the same lemma.

The proof is the same as in 4.3, except that one uses now the fact that $\sigma_{p} \beta_{p}^{R} c_{p}^{R}$ is an element of the free Abelian group $C_{p}^{S}$ (see 3.6).

4.5. Lеммa. Let $\left\{G_{p}^{(1)}\right\}, \cdots,\left\{G_{p}^{(n)}\right\}$ be identifiers for $R$, satisfying the assumptions of Lemma 4.1. Then $\left\{G_{p}^{(1)}+\cdots+G_{p}^{(n)}\right\}$ is again an identifier which satisfies the assumptions of Lemma 4.1.

The proof is obvious.

4.6. Lemma. Let $\Omega^{\prime}$ be a collection (perhaps empty) of identifiers for $R$, each of which satisfies the assumptions of Lemma 4.1. Let $\Omega$ " be a nonempty collection of identifiers for $R$, each of which satisfies the assumptions of Lemma 4.2. For each $p$, let $G_{p}$ denote the smallest subgroup of $C_{p}^{R}$ containing the groups, with the same subscript $p$, of the identifiers contained in $\Omega^{\prime \prime}$ and $\Omega^{\prime \prime}$. Then $\left\{G_{p}\right\}$ is an identifier satisfying the assumptions of Lemma 4.2.

The proof is obvious.

4.7. The preceding lemmas, combined with the results of $\S 3$, yield a number of unessential identifiers for $R$. In the following two theorems, the symbols $A_{p}^{R}$, $D_{p}^{R}, T_{p}^{R}$ have the meanings explained in $\S 3$. 
THE OREM 1. Each one of the systems $\left\{A_{p}^{R}\right\},\left\{D_{p}^{R}\right\},\left\{T_{p}^{R}\right\},\left\{A_{p}^{R}+D_{p}^{R}\right\}$, $\left\{A_{p}^{R}+T_{p}^{R}\right\},\left\{D_{p}^{R}+T_{p}^{R}\right\},\left\{A_{p}^{R}+D_{p}^{R}+T_{p}^{R}\right\}$ is an unessential identifier for $R$ (see 1.2).

THE OREM 2. If $\hat{\Gamma}_{p}^{R}$ denotes the division-hull of the group $\Gamma_{p}^{R}=A_{p}^{R}+D_{p}^{R}+T_{p}^{R}$, then $\left\{\hat{\Gamma}_{p}^{R}\right\}$ is an unessential identifier for $R$.

Proof. By 3.5 and 3.4, the system $\left\{A_{p}^{R}\right\}$ is an identifier satisfying the as sumptions of Lemma 4.2. Similarly, the systems $\left\{D_{p}^{R}\right\},\left\{T_{p}^{R}\right\}$ are identifiers satisfying the assumptions of Lemma 4.1, by 3.2 and 3.3 respectively. By 4.5 it follows then that $\left\{D_{p}^{R}+T_{p}^{R}\right\}$ is an identifier satisfying the assumptions of Lemma 4.1. Similarly, by 4.6 it follows that $\left\{A_{p}^{R}+D_{p}^{R}+T_{p}^{R}\right\},\left\{A_{p}^{R}+D_{p}^{R}\right\},\left\{A_{p}^{R}+T_{p}^{R}\right\}$ are identifiers satisfying the assumption of Lemma 4.2. Finally, $\left\{\hat{\Gamma}_{p}^{R}\right\}$ is an identifier satisfying the assumptions of Lemma 4.2 , as a consequence of 4.4 . The unessential character of all these identifiers is then a direct consequence of 4.1 and 4.2 respectively.

REMARK. The writer was unable to determine whether or not $\Gamma_{p}^{R}$ coincides with $\hat{\Gamma}_{p}^{R}$.

\section{The Complex $r=r(X)$}

5.1. Theorem 1 in 4.7 shows that any combination of the basic identification schemes, used in previous approaches to singular homology theory, may be applied to the singular complex $R$ without affecting its homology structure. From the point of view of achieving maximum reduction, the identifier $\left\{\hat{\Gamma}_{p}^{R}\right\}$ is of special interest. We shall therefore go into some detail concerning this particular identifier. By the general remarks made in $\S 1$, this identifier leads from the singular complex $R$ to a new and much smaller Mayer complex which we shall denote by $r=r(X)$. Since $\left\{\hat{\Gamma}_{p}^{R}\right\}$ is unessential, $r$ has the same homology structure as $R$. We want to examine in some detail the computational facilities and conveniences available in the complex $r$.

5.2. By the general remarks in $\$ 1$, the elements of the $p$-chain group $C_{p}^{r}$ of $r$ are of the form $\left\{c_{p}^{R}\right\}$, where this symbol denotes the coset (relative to $\hat{\Gamma}_{p}^{R}$ ) containing the $p$-chain $c_{p}^{R}$ of $R$. Let us adopt, in dealing with the complex $r$, the usual practice of writing $c_{p}^{R}$ instead of $\left\{c_{p}^{R}\right\}$, with the understanding that $c_{p}^{R}$ is now considered as a representative of the element $\left\{c_{p}^{R}\right\}$ of $C_{p}^{r}$. For clarity, we shall use the congruence symbol $\equiv$ in writing equations, to remind ourselves of the 
fact that we are dealing actually with congruences $\bmod \hat{\Gamma}_{p}^{R}$. We shall presently note some of the computational rules for the complex $r$.

5.3. Let $\left(v_{0}^{\prime}, \cdots, v_{p}^{\prime} ; T^{\prime}\right)^{R},\left(v_{0}^{\prime \prime}, \cdots, v_{p}^{\prime \prime}, T^{\prime \prime}\right)^{R}$ be two $p$-cells of $R$ related as follows. There exists a system of linearly independent points $w_{0}, \cdots, w_{p}$ in $E_{\infty}$ and two linear maps $\alpha^{\prime}:\left|w_{0}, \cdots, w_{p}\right| \rightarrow\left|v_{0}^{\prime}, \cdots, v_{p}^{\prime}\right|, \alpha^{\prime \prime}: \mid w_{0}$, $\cdots, w_{p}|\rightarrow| v_{0}^{\prime \prime}, \cdots, v_{p}^{\prime \prime} \mid$, such that the following relations hold:

$$
\begin{array}{ll}
\alpha^{\prime}\left(w_{i}\right)=v_{i}^{\prime}, \quad \alpha^{\prime \prime}\left(w_{i}\right)=v_{i}^{\prime \prime} & (i=0, \cdots, p), \\
T^{\prime} \alpha^{\prime}=T^{\prime \prime} \alpha^{\prime \prime} . &
\end{array}
$$

Then $\left(v_{0}^{\prime}, \cdots, v_{p}^{\prime}, T^{\prime}\right)^{R} \equiv\left(v_{0}^{\prime \prime}, \cdots, v_{p}^{\prime \prime}, T^{\prime \prime}\right)^{R}$. Indeed, by the definition of $A_{p}^{R}$ and $\hat{\Gamma}_{p}^{R}($ see $3.4,4.7)$, we have

$$
\left(v_{0}^{\prime}, \cdots, v_{p}^{\prime}, T^{\prime}\right)^{R}-\left(w_{0}, \cdots, w_{p}, T^{\prime} \alpha^{\prime}\right)^{R} \in A_{p}^{R} \subset \hat{\Gamma}_{p}^{R},
$$

and hence

$$
\left(v_{0}^{\prime}, \cdots, v_{p}^{\prime}, T^{\prime}\right)^{R} \equiv\left(w_{0}, \cdots, w_{p}, T^{\prime} \alpha^{\prime}\right)^{R}
$$

Similarly

$$
\left(v_{0}^{\prime \prime}, \because \cdot v_{p}^{\prime \prime}, T^{\prime \prime}\right)^{R} \equiv\left(w_{0}, \cdots, w_{p}, T^{\prime \prime} \alpha^{\prime \prime}\right)^{R} .
$$

Since $T^{\prime} \alpha^{\prime}=T^{\prime \prime} \alpha^{\prime \prime}$, the assertion follows.

5.4. Given a sequence $v_{0}, \cdots, v_{p}$ of $p+1$ points in $E_{\infty}$ (which need not be linearly independent or distinct), by a transposition we shall mean (as in $\$ 2$ ) the operation of exchanging two adjacent elements of the sequence $v_{0}, \cdots, v_{p}$. Let then $\left(v_{0}^{\prime}, \cdots, v_{p}^{\prime}, T^{\prime}\right)^{R},\left(v_{0}^{\prime \prime}, \cdots, v_{p}^{\prime \prime}, T^{\prime \prime}\right)^{R}$ be two $p$-cells related as follows:

(i) $\left|v_{0}^{\prime}, \cdots, v_{p}^{\prime}\right|=\left|v_{0}^{\prime \prime}, \cdots, v_{p}^{\prime \prime}\right|$, and $T^{\prime}=T^{\prime \prime}$;

(ii) there exists a sequence of $n \geq 0$ transpositions leading from $\left(v_{0}^{\prime}, \cdots, v_{p}^{\prime}\right)$ to $\left(v_{0}^{\prime \prime}, \cdots, v_{p}^{\prime \prime}\right)$.

Then $\left(v_{0}^{\prime}, \cdots, v_{p}^{\prime}, T^{\prime}\right)^{R} \equiv\left(v_{0}^{\prime \prime}, \cdots, v_{p}^{\prime \prime}, T^{\prime \prime}\right)^{R}$ if $n$ is even, and $\left(v_{0}^{\prime}, \cdots\right.$, $\left.v_{p}^{\prime}, T^{\prime}\right)^{R} \equiv-\left(v_{0}^{\prime \prime}, \cdots, v_{p}^{\prime \prime}, T^{\prime \prime}\right)^{R}$ if $n$ is odd. Indeed, the assertion is obvious if $n=0$. If $n=1$, the assertion follows immediately from the fact that $T_{p}^{R} \subset \hat{\Gamma}_{p}^{R}$ (see 3.2, 4.7). Repeated application of this remark yields the desired result for a general $n$. 
5.5. Let $\left(v_{0}, \cdots, v_{p}, T\right)^{R}$ be a $p$-cell such that the points $v_{0}, \cdots, v_{p}$ are not all distinct. Then $\left(v_{0}, \cdots, v_{p}, T\right)^{R} \equiv 0$. Indeed, by a certain number $n$ of transpositions we can obtain a $p$-cell $\left(w_{0}, \cdots, w_{p}, T\right)^{R}$ in which two adjacent points $w_{j}, w_{j+1}$ coincide. Then (see $3.3,4.7$ )

$$
\left(w_{0}, \cdots, w_{p}, T\right)^{R} \in D_{p}^{R} \subset \hat{\Gamma}_{p}^{R},
$$

and hence

$$
\left(w_{0}, \cdots, w_{p}, T\right)^{R} \equiv 0
$$

On the other hand, by 5.4 ,

$$
\left(w_{0}, \cdots, w_{p}, T\right)^{R} \equiv \pm\left(v_{0}, \cdots, v_{p}, T\right)^{R},
$$

and the assertion follows.

5.6. Let $\left(v_{0}, \cdots, v_{p}, T\right)^{R}$ be a p-cell of $R$. Let $w_{0}, \cdots, w_{q}$, where $q>p$, be a system of linearly independent points in $E_{\infty}$, and let $\alpha:\left|w_{0}, \cdots, w_{q}\right|$ $\rightarrow\left|v_{0}, \cdots, v_{p}\right|$ be a linear map such that the points $\alpha\left(w_{0}\right), \cdots, \alpha\left(w_{q}\right)$ coincide with the points $v_{0}, \cdots, v_{p}$ in any order and with any number of repetitions. Then

$$
\left(w_{0}, \cdots, w_{q}, T \alpha\right)^{R} \equiv 0 .
$$

Indeed, by 5.3 we have the relation

$$
\left(w_{0}, \cdots, w_{q}, T \alpha\right)^{R} \equiv\left(\alpha\left(w_{0}\right), \cdots, \alpha\left(w_{q}\right), T\right)^{R} .
$$

On the other hand, since $q>p$, the points $\alpha\left(w_{0}\right), \cdots, \alpha\left(w_{q}\right)$ are not all distinct. Hence, by 5.5 , we have

$$
\left(\alpha\left(w_{0}\right), \cdots, \alpha\left(w_{q}\right), T\right)^{R} \equiv 0,
$$

and the assertion follows.

5.7. Let $\left(v_{0}, \cdots, v_{p}, T\right)^{R}$ be a p-cell of $R$, such that the points $v_{0}, \cdots, v_{p}$ are linearly independent. Suppose this $p$-cell possesses the following type of symmetry. There exists a linear map $\alpha:\left|v_{0}, \cdots, v_{p}\right| \rightarrow\left|v_{0}, \cdots, v_{p}\right|$, such that (i) the points $\alpha\left(v_{0}\right), \cdots, \alpha\left(v_{p}\right)$ form an odd permutation of the points $v_{0}$, $\cdots, v_{p}$ (taken in the indicated order) and (ii) $T \alpha=T$. Then $\left(v_{0}, \cdots, v_{p}, T\right)^{R}$ $\equiv 0$. Indeed by 5.4 and 5.3 we have 


$$
\begin{aligned}
& \left(v_{0}, \cdots, v_{p}^{\prime}, T\right)^{R} \equiv-\left(\alpha\left(v_{0}\right), \cdots, \alpha\left(v_{p}\right), T\right)^{R}, \\
& \left(v_{0}, \cdots, v_{p}, T \alpha\right)^{R} \equiv\left(\alpha\left(v_{0}\right), \cdots, \alpha\left(v_{p}\right), T\right)^{R} .
\end{aligned}
$$

Since $T=T \alpha$, it follows that $2\left(v_{0}, \cdots, v_{p}, T\right)^{R} \equiv 0$, or equivalently

$$
2\left(v_{0}, \cdots, v_{p}, T\right)^{R} \in \hat{\Gamma}_{p}^{R} .
$$

Now since $\hat{\Gamma}_{p}^{R}$ is the division-hull of $\Gamma_{p}^{R}$ (see 4.7), the last relation implies the existence of an integer $k \neq 0$ such that $2 k\left(v_{0}, \cdots, v_{p}, T\right)^{R} \in \Gamma_{p}^{R}$, and hence (by the definition of the division-hull) $\left(v_{0}, \cdots, v_{p}, T\right)^{R} \in \hat{\Gamma}_{p}^{R}$. Thus $\left(v_{0}, \cdots\right.$, $\left.v_{p}, T\right)^{R} \equiv 0$.

5.8. The argument just used yields obviously the general result: if $n c_{p}^{R} \equiv 0$, where $n$ is an integer $\neq 0$, then $c_{p}^{R} \equiv 0$. In other words, the p-chain group $C_{p}^{r}$ of the complex $r$ has no elements of finite order. Of course, this is a priori obvious from the remark that a division-hull is closed under division. It may be of interest to determine whether or not $C_{p}^{r}$ is in fact a free Abelian group. The writer was unable to answer this question.

5.9. The homomorphisms $\partial_{p}^{R}, \beta_{p}^{R}, \rho_{p}^{R}, \tau_{p} \sigma_{p}$ apply to congruences. In detail: if $c_{p}^{R} \equiv \bar{c}_{p}^{R}$, then

$$
\partial_{p}^{R} c_{p}^{R} \equiv \partial_{p}^{R} \bar{c}_{p}^{R}, \quad \beta_{p}^{R} c_{p}^{R} \equiv \beta_{p}^{R} \bar{c}_{p}^{R}, \quad \rho_{p}^{R} c_{p}^{R} \equiv \rho_{p}^{R} \bar{c}_{p}^{R}, \quad \tau_{p} \sigma_{p} c_{p}^{R} \equiv \tau_{p} \sigma_{p} \bar{c}_{p}^{R} .
$$

The first one of these asserted congruences is of course merely a restatement of the fact that $\left\{\hat{\Gamma}_{p}^{R}\right\}$ is an identifier. The last one may be verified as follows. In view of the identity 3.1 (4) we have

$$
\sigma_{p}\left(\tau_{p} \sigma_{p} c_{p}^{R}-c_{p}^{R}\right)=\sigma_{p} c_{p}^{R}-\sigma_{p} c_{p}^{R}=0,
$$

and hence, by 3.4 ,

$$
\tau_{p} \sigma_{p} c_{p}^{R}-c_{p}^{R} \in A_{p}^{R}
$$

Since $A_{p}^{R} \subset \hat{\Gamma}_{p}^{R}$, it follows that

$$
\tau_{p} \sigma_{p} c_{p}^{R} \equiv c_{p}^{R}
$$

Similarly, $\tau_{p} \sigma_{p} \bar{c}_{p}^{R} \equiv \bar{c}_{p}^{R}$. Since $c_{p}^{R} \equiv \bar{c}_{p}^{R}$, it follows that $\tau_{p} \sigma_{p} c_{p}^{R} \equiv \tau_{p} \sigma_{p} \bar{c}_{p}^{R}$. Now let us recall that $\left\{\hat{\Gamma}_{p}^{R}\right\}$ satisfies the assumptions of Lemma 4.2 , as we observed in the course of the proof in 4.7. Accordingly, the assumption $c_{p}^{R} \equiv \bar{c}_{p}^{R}$, which is equivalent to $c_{p}^{R}-\bar{c}_{p}^{R} \in \hat{\Gamma}_{p}^{R}$, implies that 


$$
\begin{aligned}
& \sigma_{p} \beta_{p}^{R}\left(c_{p}^{R}-\bar{c}_{p}^{R}\right)=0, \\
& \rho_{p}^{R}\left(c_{p}^{R}-\bar{c}_{p}^{R}\right) \in \hat{\Gamma}_{p+1}^{R} .
\end{aligned}
$$

The relation (3) is equivalent to $\rho_{p}^{R} c_{p}^{R} \equiv \rho_{p}^{R} c_{p}^{R}$. On the other hand, (2) implies, by 3.4, that $\beta_{p}^{R}\left(c_{p}^{R}-\bar{c}_{p}^{R}\right) \in A_{p}^{R} \subset \hat{\Gamma}_{p}^{R}$, and hence that $\beta_{p}^{R} c_{p}^{R} \equiv \beta_{p}^{R} \bar{c}_{p}^{R}$.

5.10. In terms of familiar terminology, the preceding results may be summarized as follows. In the complex $r$, affine-equivalent $p$-cells of $R$ become equal to each other (see 5.3). The permutation rule (or the orientation convention) holds in $r$ (see 5.4). Degenerate p-cells of $R$ may be discarded in $r$ (see 5.5, 5.6), as well as affine-symmetric $p$-cells (see 5.7). The operators $\partial_{p}^{R}, \beta_{p}^{R}, \rho_{p}^{R}$ continue to apply in $r$ (see 5.9). Furthermore, the operation $\tau_{p} \sigma_{p}$ is also applicable in $r$ (see 5.9). The effect of this operation is to replace a general $p$-cell $\left(v_{0}, \cdots, v_{p}, T\right)^{R}$ by a $p$-cell of the form $\left(d_{0}, \cdots, d_{p}, T^{*}\right)$ (see 0.3$)$. Accordingly, one can avoid entirely the use of $p$-cells $\left(v_{0}, \cdots, v_{p}, T\right)^{R}$ where the points $v_{0}, \cdots, v_{p}$ are not linearly independent (it is not obvious, however, that this practice, if followed consistently, contributes to clarity and simplicity of calculations). Finally, let us note that the complex $r$ offers the advantage that its chain-groups do not have elements of finite order (see 5.8). In the light of comments made in previous literature, this may represent a desirable feature.

5.11. In the course of a correspondence on these subjects, Professor S. MacLane communicated to the writer a simple and ingenious proof of the fact that the chain-groups of the complex $r$ are indeed free Abelian groups (cf. 5.8).

\section{CoNCLUSION}

6.1. One may raise the question whether the singular complex $R$ admits of further reductions, in terms of identifications, without affecting its homology structure. In particular, one may ask whether there exists a maximal identification scheme, in some natural and appropriate sense. A plausible approach may be obtained by setting up the principle that only those identifications are admitted for which the computational rules set forth in 5.3-5.9 hold. The problem consists then of determining whether among all unessential identifiers $\left\{G_{p}\right\}$, conforming to this principle, there exists one, say $\left\{\bar{G}_{p}\right\}$, such that $G_{p} \subset \bar{G}_{p}$ for all identifiers $\left\{G_{p}\right\}$ satisfying the requirements just stated. The writer was unable to settle various interesting questions upon which the answer to this problem seems to depend.

6.2. From a heuristic point of view, one may conjecture that, in view of the intensive study and manifold applications of singular homology theory, it is unlikely that any relevant identification scheme escaped the attention of the many 
workers in this field. For example, one may assume, as a heuristic working hypothesis, that by applying simultaneously all the identification schemes used in the papers listed in the References of the present paper one obtains a maximal identification scheme in the sense of 6.1. The writer was unable to find a proof for the theorem suggested by these remarks.

6.3. As regards previous literature concerned with the unessential character of identification schemes, precise comparisons would lead to excessive detail, particularly because our complex $R$ has not been considered explicitly in the literature, as far as the writer is aware. The following comments are meant to indicate the origin of certain questions rather than the exact formulation of definitions occurring in other theories. The initial motivation for the present study, as well as for the previous paper [6] of the writer, came from the important paper of Eilenberg [1]. In that paper, Eilenberg shows, in effect, that (in our terminology) the identifier $\left\{T_{p}^{R}\right\}$ is unessential (see 3.2). In his previous paper [6], the writer showed then that the identifier $\left\{A_{p}^{R}\right\}$ is also unessential. However, the unessential character of certain identifications has been recognized by various authors. Thus Seifert-Threlfall [7] and Lefschetz [5] contain remarks suggesting that the "affine symmetric" p-cells may be discarded without affecting the homology structure. Tucker [8] showed, in effect, that the system $\left\{D_{p}^{R}\right\}$ is unessential, at least in relation to the identifier $\left\{T_{p}^{R}\right\}$. In a sense, our complex $R$ appears thus as the singular complex in unreduced form, alternative theories being derivable by various types of reduction. The problems we stated in 6.1 and 6.2 amount merely to the question whether there is some end to this process of reduction without changing the homology structure.

\section{REFERENCES}

1. S. Eilenberg, Singular homology theory, Ann. of Math. 45 (1944), 407-447.

2. S. Eilenberg and N. E. Steenrod, Foundations of Algebraic Topology (Unpublished).

3. S. Eilenberg and J. A. Zilber, Semi-simplicial complexes and singular homology, Ann. of Math. 51 (1950), 499-513.

4. S. Lefschetz, Algebraic Topology, Amer. Math. Soc. Colloquium Publications, vol. 27; American Mathematical Society, New York, 1942.

5. - On singular chains and cycles, Bull. Amer. Math. Soc. 39 (1933), 124-129.

6. T. Radó, On identifications in singular homology theory, To appear in Rivista di Matematica della Università di Parma.

7. H. Seifert and W. Threlfall, Lehrbuch der Topologie, B. G. Teubner, Leipzig, 1934.

8. A. W. Tucker, Degenerate cycles bound, Rec. Math. (Mat. Sbornik) 3 (1938), 287-289.

\section{Ohio State University}




\title{
PACIFIC JOURNAL OF MATHEMATICS
}

\section{EDITORS}

\author{
Herbert BuSEMANN \\ R. M. RoBINSON \\ University of Southern California \\ University of California \\ Los Angeles 7, California \\ Berkeley 4, California \\ E. F. BEC KENBACH, Managing Editor \\ University of California \\ Los Angeles 24, California
}

\section{ASSOCIATE EDITORS}
R. P. DILWORTH
P. R. HALMOS
BØRGE JESSEN
J. J. STOKER
HERBERT FEDERER
HEINZ HOPF
PAUL LÉVY
MARSHALL HALL
R. D. JAMES
GEORGE PÓLYA
E. G. STRAUS
KÖSAKU YOSIDA

\section{SPONSORS}

UNIVERSITY OF BRITISH COLUMBIA

CALIFORNIA INSTITUTE OF TECHNOLOGY

UNIVERSITY OF CALIFORNIA, BERKELEY

UNIVERSITY OF CALIFORNIA, DAVIS

UNIVERSITY OF CALIFORNIA, LOS ANGELES

UNIVERSITY OF CALIFORNIA, SANTA BARBARA

OREGON STATE COLLEGE

UNIVERSITY OF OREGON
UNIVERSITY OF SOUTHERN CALIFORNIA

STANFORD UNIVERSITY

WASHINGTON STATE COLLEGE

UNIVERSITY OF WASHINGTON

AMERICAN MATHEMATICAL SOCIETY

NATIONAL BUREAU OF STANDARDS, INSTITUTE FOR NUMERIGAL ANALYSIS

Mathematical papers intended for publication in the Pacific Journal of Mathematics should be typewritten (double spaced), and the author should keep a complete copy. Manuscripts may be sent to any of the editors. All other communications to the editors should be addressed to the managing editor, E. F. Beckenbach, at the address given above.

Authors are entitled to receive 100 free reprints of their published papers and may obtain additional copies at cost.

The Pacific Journal of Mathematics is published quarterly, in March, June, September, and December. The price per volume (4 numbers) is $\$ 8.00$; single issues, $\$ 2.50$. Spécial price to individual faculty members of supporting institutions and to members of the American Mathematical Society: $\$ 4.00$ per volume; single issues, $\$ 1.25$.

Subscriptions, orders for back numbers, and changes of address should be sent to the publishers, University of California Press, Berkeley 4, California.

UNIVERSITY OF CALIFORNIA PRESS - BERKELEY AND LOS ANGELES 


\section{Pacific Journal of Mathematics}

\section{Vol. 1, No. $2 \quad$ December, 1951}

Tom M. (Mike) Apostol, On the Lerch zeta function ................. 161

Ross A. Beaumont and Herbert S. Zuckerman, A characterization of the subgroups of the additive rationals ....................... 169

Richard Bellman and Theodore Edward Harris, Recurrence times for the Ehrenfest model................................... 179

Stephen P.L. Diliberto and Ernst Gabor Straus, On the approximation of a function of several variables by the sum of functions of fewer

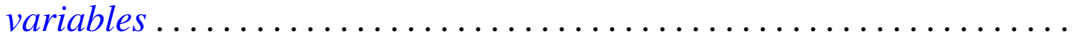

Isidore Isaac Hirschman, Jr. and D. V. Widder, Convolution transforms with complex kernels ................................ 211

Irving Kaplansky, A theorem on rings of operators .............. 227

W. Karush, An iterative method for finding characteristic vectors of a symmetric matrix............................... 233

Henry B. Mann, On the number of integers in the sum of two sets of positive integers ......................................... 249

William H. Mills, A theorem on the representation theory of Jordan

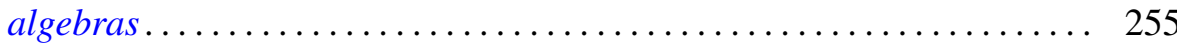

Tibor Radó, An approach to singular homology theory.............. 265

Otto Szász, On some trigonometric transforms ................... 291

James G. Wendel, On isometric isomorphism of group algebras ......... 305

George Milton Wing, On the $L^{p}$ theory of Hankel transforms ... 